\title{
Uma abordagem dos ensaios in vitro para estimar a absorção dos minerais em fórmulas infantis
}

\section{An in vitro testing approach to estimate mineral absorption in infant formulas}

\author{
Maria Isabel Andrekowisk Fioravanti ${ }^{1,2 *}$ (D), Marcelo Antônio Morgano² (D) \\ ${ }^{1}$ Instituto Adolfo Lutz, Campinas/SP - Brasil \\ ${ }^{2}$ Instituto de Tecnologia de Alimentos, Campinas/SP - Brasil
}

*Corresponding author: Maria Isabel Andrekowisk Fioravanti, Instituto Adolfo Lutz, Rua São Carlos, 720, Vila Industrial, CEP: 13035-420, Campinas/SP - Brasil, e-mail: maria.fioravanti@ial.sp.gov.br

Cite as: Fioravanti, M. I. A., \& Morgano, M. A. (2021). An in vitro testing approach to estimate mineral absorption in infant formulas. Brazilian Journal of Food Technology, 24, e2020098. https://doi.org/10.1590/1981-6723.09820

\begin{abstract}
Resumo
A fórmula infantil é a opção de alimento mais recomendada quando a amamentação não é possível. Para o melhor entendimento do comportamento gastrointestinal e da absorção dos nutrientes presentes nas fórmulas infantis, realizam-se estudos que simulam os processos de digestão. Este trabalho teve como objetivo discutir a evolução das fórmulas infantis com foco na avaliação da absorção de $\mathrm{Ca}, \mathrm{Cu}, \mathrm{Fe}, \mathrm{K}, \mathrm{Mg}, \mathrm{Mn}, \mathrm{P}$ e Zn, identificando métodos disponíveis para a avaliação de bioacessibilidade e de biodisponibilidade, de forma a melhor entender a absorção desses nutrientes. Para isto, foi realizada uma revisão bibliográfica sobre fórmulas infantis e os métodos in vitro mais utilizados no estudo de minerais, com foco no consumo, na qualidade e nos aspectos tecnológicos. Foi constatada uma tendência mundial no aumento do consumo de fórmulas infantis e verificou-se que estudos de biodisponibilidade e bioacessibilidade de minerais, mesmo que ainda escassos, colaboram para a evolução e o desenvolvimento deste produto, visando a uma aproximação com a composição do leite materno. A pesquisa mostrou que a composição da fórmula infantil vem sendo constantemente aperfeiçoada por revisões das legislaç̃̃es e pela indústria, e que, para a obtenção de uma fórmula infantil com alta capacidade de absorção de nutrientes, devem ser realizados estudos de bioacessibilidade e/ou de biodisponibilidade, pois estes estudos possibilitam uma melhor compreensão da influência dos diferentes ingredientes na absorção dos nutrientes. Os estudos também mostraram que, para uma ingestão mais equilibrada, nas diferentes fases do lactente, são necessárias diferentes composições dos nutrientes nas fórmulas infantis.
\end{abstract}

Palavras-chave: Biodisponibilidade; Nutrição do lactente; Alimento infantil; Micronutrientes; Segurança dos alimentos; Ingestão.

\begin{abstract}
Infant formula is the best alternative food for providing nutrition to the infant, when breastfeeding is not possible. For a better understanding of gastrointestinal behavior and absorption of nutrients present in infant formulas, studies that simulate digestion processes may be used. This study aimed to discuss the evolution of infant formulas
\end{abstract}


with a focus on the evaluation of the absorption of $\mathrm{Ca}, \mathrm{Cu}, \mathrm{Fe}, \mathrm{K}, \mathrm{Mg}, \mathrm{Mn}, \mathrm{P}$ and $\mathrm{Zn}$, as well as identifying available methods for the assessment of bioaccessibility and bioavailability, in order to understand the absorption of these nutrients. For this purpose, a review was carried out about infant formulas and also about in vitro methods regarding the study of minerals, being focused on consumption, quality and technological aspects. A worldwide trend was observed in the increase concerning the consumption of infant formulas and it could be noted that studies of bioavailability and bioaccessibility of minerals, even if these studies are still scarce, collaborate for the evolution and development of this product, aiming to approximate the composition of breast milk. The review showed that the composition of the infant formula has been constantly improved by revisions of legislation and by the industry and, in order to obtain an infant formula with a high nutrient absorption capacity, bioaccessibility and / or bioavailability studies must be carried out, because these studies allow a better understanding of the influence of different ingredients on the absorption of nutrients. These studies have also shown that for making up a more balanced intake in the different stages of the infant, different compositions of infant formulas are required.

Keywords: Bioavailability; Infant nutrition; Infant food; Micronutrients; Food safety; Ingestion.

\section{Introdução}

Quando a amamentação não é possível, desejável ou as mães são incapazes de fornecer leite suficiente, fontes alternativas balanceadas de nutrientes devem ser usadas. Atualmente, a opção recomendada para crianças nascidas a termo saudáveis (recém-nascido cuja idade gestacional foi entre 37 semanas e 41 semanas e 6 dias) é a fórmula infantil (FI) (Silva et al., 2013b; Martin et al., 2016; Zou et al., 2016).

Uma ingestão equilibrada de nutrientes do lactente pode ser conseguida pelo consumo de FI e é, sem dúvida, mais adequada do que o consumo de leite de vaca (Food and Agriculture Organization of the United Nations, 2016). O consumo de minerais durante a primeira infância é um fator crítico devido à alta taxa de crescimento das crianças e sua deficiência pode afetar o desenvolvimento e gerar consequências ao longo de sua vida (Silva et al., 2013b).

A determinação do teor total dos nutrientes presentes em FI é fundamental para garantir a qualidade do produto, segundo as legislações e normas (Brasil, 2011a). Todavia, conhecer a concentração total de nutrientes no alimento não é suficiente para determinar se o alimento provê as necessidades nutricionais adequadas, sendo importante o conhecimento da fração que pode estar efetivamente disponível para absorção pelo organismo (Silva et al., 2013a). Neste sentido, é importante o estudo da bioacessibilidade ou da biodisponibilidade dos nutrientes, de forma a avaliar sua potencial absorção pelo organismo (Minekus et al., 2014).

Os métodos in vitro que simulam os processos de digestão são amplamente utilizados para avaliar o comportamento dos alimentos no trato gastrointestinal e a absorção de nutrientes. Em comparação aos ensaios in vivo, os métodos in vitro apresentam as vantagens de serem mais rápidos, menos dispendiosos e trabalhosos, sem restrições éticas, além de permitirem que um número relativamente grande de amostras seja medido para fins de triagem (Minekus et al., 2014). Assim, o objetivo deste trabalho foi realizar uma revisão sobre a evolução das FI com foco na avaliação da absorção de minerais ( $\mathrm{Ca}, \mathrm{Cu}, \mathrm{Fe}, \mathrm{K}, \mathrm{Mg}, \mathrm{Mn}, \mathrm{P}$ e $\mathrm{Zn}$ ), além de identificar os estudos envolvendo métodos in vitro que contribuem para um melhor entendimento da absorção destes elementos.

\section{Método}

O trabalho foi realizado por meio de uma revisão bibliográfica sobre FI e métodos de bioacessibilidade e de biodisponibilidade in vitro, para estudo de minerais. Foram pesquisados documentos científicos atualizados nas bases de dados do Portal de Periódicos da Coordenação de Aperfeiçoamento de Pessoal de Nível Superior (Capes), PubMed, Scientific Electronic Library (SciELO), Scopus, Web of Science e Google Acadêmico, usando as seguintes palavras-chave: fórmula infantil, bioacessibilidade, biodisponibilidade e 
minerais. Visando a uma maior abrangência sobre o tema, também foram realizadas buscas em legislações e sites institucionais, para buscar teses e dissertações sobre o tema. Duas perguntas foram norteadoras do trabalho: 1) Como se comportam as normas e o consumo de FI? e 2) $O$ que os estudos com ensaios in vitro estão apontando sobre a absorção dos minerais em FI?

A busca ocorreu entre o período de março de 2018 e dezembro de 2019, e os critérios de inclusão e exclusão dos materiais foram: incluíram-se artigos completos publicados em revistas indexadas; não houve restrição quanto à data de publicação; excluíram-se textos que abordavam FI e métodos in vitro não relacionados aos minerais. Em relação às legislações, foram utilizadas todas em vigor sobre a matriz FI, nacionais e internacionais, além do Codex Alimentarius Standard for Infant Formula.

\section{Fórmulas infantis}

\subsection{Fórmulas infantis como substituto do leite materno}

Segundo o Codex Alimentarius Standard for Infant Formula, a FI é um substituto do leite materno especialmente preparado para satisfazer, por si só, os requisitos nutricionais dos bebês durante os primeiros meses de vida até a introdução da alimentação complementar adequada. A segurança nutricional e a adequação das fórmulas para lactentes devem ser cientificamente demonstradas para apoiar o crescimento e o desenvolvimento de lactentes (Food and Agriculture Organization of the United Nations, 2016).

Globalmente, apenas 38\% das crianças são exclusivamente amamentadas até os seis meses de idade (Martin et al., 2016). Um estudo realizado, entre 2012 e 2015, com 700 lactentes (idade mediana de 5,5 meses), na cidade de São Paulo, sobre a introdução de alimentos complementares, demonstrou que a água (80\%), seguida pela FI (64\%) e pelo suco (51\%), foram os alimentos mais utilizados e mais precocemente introduzidos aos lactentes (Moreira et al., 2019). O enfoque deste estudo mostra como os conceitos, padrões e práticas relacionados a qualidade e segurança dos produtos alimentícios voltados para lactentes são de grande importância para a saúde pública (Donovan, 2019).

As FI disponíveis hoje são classificadas de acordo com a faixa etária do lactente e incluem, entre outras denominações: "FI para lactentes", para os primeiros seis meses de vida; "FI de seguimento para lactentes", a partir do sexto mês de vida até doze meses de idade incompletos; "FI para lactentes e de seguimento para lactente", para os primeiros doze meses de idade incompletos, e "FI para crianças de primeira infância", para crianças de 1 a 3 anos. Existe também a categoria de FI para necessidades dietoterápicas específicas, que atendem às necessidades nutricionais decorrentes de alterações fisiológicas, de doenças temporárias ou permanentes, e de risco de alergias, bem como para recém-nascido pré-termo (prematuro com menos de 37 semanas de estado gestacional) (European Commission, 2006; Brasil, 2011b; Brasil, 2011c; Brasil, 2011d).

\subsection{Legislação para fórmulas infantis}

Atualmente, a segurança da FI é garantida por rigorosas legislações, por um controle de qualidade que emprega métodos analíticos bem estabelecidos e por novos estudos que trazem soluções práticas para minimizar possíveis incidentes, principalmente os microbiológicos. Estes incidentes podem afetar as características organolépticas, influenciando o consumidor e diminuindo a credibilidade em relação à qualidade do produto (Fei et al., 2020; Fioravanti et al., 2020a; Fu et al., 2020; Pires et al., 2020; Li et al., 2019). Para prevenir cada vez mais estes incidentes, os regulamentos técnicos de FI para lactentes e produtos lácteos são delineados por padrões internacionais elaborados pela Comissão do Codex Alimentarius ou por padrões específicos elaborados principalmente pela União Europeia e pelos seguintes países: Estados Unidos, China, Austrália e Nova Zelândia (Poitevin, 2016). A composição da FI é estritamente regulamentada e cada fabricante deve seguir as diretrizes estabelecidas pelas agências governamentais do país onde será comercializada. 
A quantidade dos compostos (nutrientes) para as FI de diferentes faixas etárias deve estar de acordo com o regulamento técnico específico, como por exemplo, os diferentes limites para o elemento $\mathrm{Fe}$, apresentados na Tabela 1. Note-se que determinados compostos podem não ser aplicáveis a alguns tipos de fórmulas, principalmente aquelas voltadas para necessidades dietoterápicas específicas, como é o caso de FI que atende a lactente com restrição de lactose, que deve conter um teor de lactose inferior ou igual a $10 \mathrm{mg} / 100 \mathrm{kcal}$ (2,5 mg/ $100 \mathrm{~kJ})$ (Brasil, 2011d).

A Tabela 1 apresenta os níveis mínimos (Min) e máximos (Max), e os limites superiores de referência (LSR) ( $\mu \mathrm{g}$ ou $\mathrm{mg} / 100 \mathrm{kcal})$ recomendados pelo Codex Alimentarius e estabelecidos pelas legislações brasileiras (RDC), europeia (UE) e americana (FDA) (Food and Agriculture Organization of the United Nations, 2016; European Commission, 2006; Brasil, 2011b; Brasil, 2011c; U.S. Food and Drug, 1985). Os LSR são estipulados para os nutrientes sem informações suficientes quanto à avaliação de risco, obtidos a partir das necessidades nutricionais dos lactentes e do histórico de uso aparentemente seguro (Food and Agriculture Organization of the United Nations, 2016; Poitevin, 2016).

Observando a Tabela 1, nota-se que a UE e o FDA adotaram apenas valores Min e Max, enquanto que o Brasil seguiu rigorosamente as recomendações do Codex Alimentarius. Os níveis para o elemento Fe são diferentes entre todas as legislações, sendo que a recomendação do Codex Alimentarius é que as autoridades de cada país estabeleçam estes valores levando em consideração as características da alimentação da população e o teor de Fe no suprimento de água.

Tabela 1. Níveis mínimos (min), máximos (max) e Limite Superior de Referência (LSR) recomendados pelo Codex Alimentarius e estabelecidos pelas legislações brasileiras, europeia e americana.

\begin{tabular}{|c|c|c|c|c|c|c|c|c|c|}
\hline \multirow{3}{*}{ Mineral } & \multirow{2}{*}{\multicolumn{2}{|c|}{ Codex 2016}} & \multirow{2}{*}{\multicolumn{2}{|c|}{$\frac{R D C n^{\circ} 43}{0-6 \text { meses }}$}} & \multirow{2}{*}{\multicolumn{2}{|c|}{$\frac{\text { RDC n44 }}{\text { 6-12 meses }}$}} & \multicolumn{2}{|c|}{ UE } & \multirow{3}{*}{$\begin{array}{c}\text { FDA } \\
\text { 0-12 meses } \\
\text { Min-Max }\end{array}$} \\
\hline & & & & & & & \multirow{2}{*}{$\begin{array}{l}\text { 0-6 meses } \\
\text { Min-Max }\end{array}$} & \multirow{2}{*}{$\begin{array}{l}\text { 6-12 meses } \\
\text { Min-Max }\end{array}$} & \\
\hline & $\begin{array}{l}\text { Min- } \\
\text { Max" }\end{array}$ & LSR & $\begin{array}{l}\text { Min- } \\
\text { Max }\end{array}$ & LSR & $\begin{array}{l}\text { Min- } \\
\text { Max }\end{array}$ & LSR & & & \\
\hline $\mathrm{Ca}(\mathrm{mg} / 100 \mathrm{kcal})$ & 50 & 140 & 50 & 140 & 50 & 140 & $50-140$ & $50-140$ & 60 \\
\hline $\mathrm{Cu}(\mu \mathrm{g} / 100 \mathrm{kcal})$ & 35 & 120 & 35 & 120 & 35 & 120 & $35-100$ & $35-100$ & 60 \\
\hline $\mathrm{Fe}(\mathrm{mg} / 100 \mathrm{kcal})$ & 0,5 & - & $0,5-1,3$ & - & $0,9-2$ & - & $0,3-1,3$ & $0,6-2$ & $0,2-3$ \\
\hline $\mathrm{K}(\mathrm{mg} / 100 \mathrm{kcal})$ & $60-180$ & - & $60-180$ & - & $60-180$ & - & $60-160$ & $60-160$ & $80-200$ \\
\hline $\operatorname{Mg}(\mathrm{mg} / 100 \mathrm{kcal})$ & 5 & 15 & 5 & 15 & 5 & 15 & $5-15$ & $5-15$ & 6 \\
\hline $\operatorname{Mn}(\mu \mathrm{g} / 100 \mathrm{kcal})$ & 1 & 100 & 1 & 100 & 1 & 100 & $1-100$ & $1-100$ & 5 \\
\hline $\mathrm{P}$ (mg / $100 \mathrm{kcal})$ & 25 & 100 & 25 & 100 & 25 & 100 & $25-90$ & $25-90$ & 30 \\
\hline $\mathrm{Zn}(\mathrm{mg} / 100 \mathrm{kcal})$ & 0,5 & 1,5 & 0,5 & 1,5 & 0,5 & 1,5 & $0,5-1,5$ & $0,5-1,5$ & 0,5 \\
\hline $\mathrm{Na}(\mathrm{mg} / 100 \mathrm{kcal})$ & $20-60$ & - & $20-60$ & - & $20-60$ & - & $20-60$ & $20-60$ & $20-60$ \\
\hline
\end{tabular}

*Min-Max: quando há apenas um valor, não há máximo estipulado, quando há dois valores (X-Y), mínimos e máximos estipulados. LSR: Limite Superior de Referência.

Fontes: Food and Agriculture Organization of the United Nations (2016); Brasil, (2011b, 2011c); European Commission (2006); U.S. Food and Drug (1985).

\subsection{Evolução dos estudos sobre nutrientes em FI}

Nos últimos 30 anos, a composição da FI vem evoluindo para se aproximar ao máximo do leite materno. Atualmente, não apenas as composições das FI estão sendo alteradas para se aproximar do leite materno, mas também novas tecnologias estão se tornando populares, melhorando a absorção dos nutrientes e garantindo uma nutrição mais balanceada para os lactentes. 
Estudos demonstraram que a incorporação de diferentes ingredientes traz benefícios às FI, como, por exemplo, a adição de ácidos graxos poli-insaturados de cadeia longa (LCPUFAs), nucleotídeos, aminoácidos essenciais e prebióticos, e o desenvolvimento de fórmulas especializadas para necessidades específicas (Zou et al., 2016). Mesmo a adição de ingredientes como a lecitina de soja, usada para reduzir o processo de oxidação do produto, às vezes também é incorporada à FI para ajudar a atender aos níveis de colina necessários (Drapala et al., 2018).

No entanto, persistem disparidades entre lactentes alimentados com leite materno e com fórmula quanto ao crescimento, ao desenvolvimento neurológico e à composição do microbioma. Aceita-se que os componentes bioativos no leite materno sejam, em parte, responsáveis por essas diferenças (Donovan, 2019). Parte da razão dessas diferenças também é atribuída à abordagem estática nas composições das FI. O leite materno é uma matriz complexa com uma composição dinâmica que muda ao longo do tempo, adaptando-se às novas necessidades da criança em crescimento. Essas mudanças naturais do leite materno não foram amplamente consideradas no desenvolvimento das FI (Lönnerdal \& Hernell, 2016, Martin et al., 2016), sendo que a exigência de diferentes faixas etárias previstas nas legislações e organizações é um esforço para minimizar as diferenças entre FI e o leite materno.

\subsection{Importância dos minerais na FI}

Estudos que quantificam os macrominerais $(\mathrm{Ca}, \mathrm{K}, \mathrm{Na}, \mathrm{P}, \mathrm{Mg})$ e os microminerais ( $\mathrm{Fe}, \mathrm{Cu}, \mathrm{Mn}, \mathrm{Zn})$ em leite materno geram dados de referência e são utilizados para estabelecer recomendações dietéticas confiáveis quanto à ingestão dos nutrientes durante a infância. Essas informações nutricionais são, portanto, a base para que a indústria de alimentos desenvolva produtos fortificados o mais próximo da composição mineral do leite materno (Zou et al., 2016).

Em geral, as FI apresentam uma concentração total de minerais maior em comparação às concentrações encontradas em leite materno. Bosscher et al. (2003) demonstraram que a alta concentração de minerais na FI não corresponde a uma maior biodisponibilidade dos elementos, já que os resultados encontrados para disponibilidades em leite materno foram de 19,6\% para $\mathrm{Ca}, 30,2 \%$ para $\mathrm{Fe}$ e $48,2 \%$ para $\mathrm{Zn}$, enquanto que, para a FI, foram de $13,5 \%$ para $\mathrm{Ca}, 3,4 \%$ para $\mathrm{Fe}$ e $9,3 \%$ para $\mathrm{Zn}$.

O estudo realizado por Martínez et al. (2019) com FI comercializadas na Espanha e leite materno demonstrou que os níveis dos minerais e elementos traço - entre eles, $\mathrm{Ca}, \mathrm{Cu}, \mathrm{Fe}, \mathrm{K}, \mathrm{Mg}, \mathrm{Mn}, \mathrm{Na}$ e $\mathrm{Zn}$ - foram significativamente maiores nas fórmulas, em relação aos verificados no leite materno. Ao observar os resultados por faixa etária, as concentrações de $\mathrm{Ca}, \mathrm{Fe}, \mathrm{K}, \mathrm{Mg}$ e Na nas FI para lactentes (0-6 meses) foram estatisticamente menores do que nas amostras de FI de seguimento para lactentes (6-12 meses). Esse é um resultado interessante, que demonstra uma tendência dos fabricantes em seguirem variações diferentes entre as faixas etárias, mesmo que a legislação da União Europeia exija diferença de concentração apenas para o Fe. Esta tendência de maior concentração dos elementos nas FI destinadas a crianças maiores de 6 meses pode estar relacionada aos diferentes níveis de ingestões diários recomendados para estas faixas etárias (Padovani et al., 2006; Zou et al., 2016).

\subsection{Bioacessibilidade de minerais em FI}

Os desenvolvimentos de modelos de digestão in vitro baseiam-se em uma extensa compreensão das condições de digestão in vivo, de diferentes grupos populacionais. A elaboração de alimentos que atendam às necessidades específicas de determinados grupos, como crianças, gestantes, idosos, diabéticos, entre outros, pode melhorar a capacidade de explorar o potencial dos alimentos, prevenir doenças e manter a saúde (Brodkorb et al., 2019; Shani-Levi et al., 2017).

A princípio, os modelos de digestão in vitro são uma alternativa aos modelos in vivo para o estudo de ingredientes alimentares, fornecendo resultados precisos em pouco tempo e servindo como uma ferramenta de triagem. Na prática, qualquer método in vitro inevitavelmente não corresponde à precisão e ao alcance dos estudos de alimentos in vivo devido à complexidade inerente ao processo digestivo humano (Hur et al., 2011). A maioria dos métodos 
desenvolvidos simula uma sequência de eventos que ocorrem durante a digestão no trato gastrointestinal humano, permitindo estimar qual porcentagem dos nutrientes estará disponível para absorção (conteúdo bioacessível) e disponível para funções fisiológicas (conteúdo biodisponível) (Alegría-Torán et al., 2015).

É importante diferenciar os termos bioacessibilidade e biodisponibilidade. A bioacessibilidade é definida como a fração de um elemento que é liberado da matriz alimentar após ingestão e solubilizado no lúmen intestinal e trato intestinal. A biodisponibilidade de um composto depende das propriedades físicas da matriz alimentar e descreve a fração do elemento solubilizado que é absorvido no trato intestinal e que atinge o sistema circulatório (BarcielaAlonso \& Bermejo-Barrera, 2015). Outros termos também usados são: ensaio de dialisabilidade, que se refere à digestão gastrointestinal seguida da determinação da quantidade do nutriente que atravessa uma membrana semipermeável, simulando a parede intestinal, e ensaio de solubilidade, que se refere à digestão gastrointestinal a partir da fração solúvel do elemento de interesse (Moreda-Piñeiro et al., 2011).

Os estudos de bioacessibilidade são importantes e auxiliam na identificação de matrizes alimentares promissoras e/ou nas condições de processamento e no impacto na biodisponibilidade dos nutrientes (Fioravanti et al., 2020b; Etcheverry et al., 2012). Dentre os alimentos mais estudados, aplicando métodos in vitro, destacam-se as hortaliças (26\%), os laticínios (23\%), os produtos de panificação (17\%), os produtos cárneos $(13 \%)$, os alimentos marinhos (12\%) e os alimentos à base de ovos (7\%) (Lucas-González et al., 2018). O número de estudos disponíveis na literatura sobre a determinação de minerais na matriz FI e estudos de bioacessibilidade de minerais são altos (PubMed: 139 artigos para "mineral in infant formula" e 229 artigos para "mineral bioaccessibility", em 01/12/2019). Porém, ao especificar na busca "estudos de bioacessibilidade e/ou biodisponibilidade de minerais na matriz FI", o número de estudos encontrado foi significativamente menor (20 artigos).

A Tabela 2 apresenta os estudos encontrados. Observe-se que, em geral, os métodos in vitro envolvem uma digestão gastrointestinal simulada com pepsina durante a etapa gástrica, seguida pela etapa intestinal com pancreatina e sais biliares para a determinação das frações solúvel e dialisada de $\mathrm{Ca}, \mathrm{Cu}, \mathrm{Fe}$ e $\mathrm{Zn}$. Dos 20 estudos apresentados, envolvendo bioacessibilidade e/ou biodisponibilidade de minerais em FI, apenas dois descreveram uma etapa oral prévia à digestão gastrointestinal (Silva et al., 2013a; Gomez et al., 2016). As referências mais citadas entre os artigos encontrados foram os estudos de Miller et al. (1981) e Luten et al. (1996), com adaptações referentes ao volume de enzimas e sais biliares adicionados durante a digestão intestinal.

Todos os estudos apresentados na Tabela 2 citam FI e métodos in vitro, contudo nem todos realizaram a avaliação da bioacessibilidade dos elementos nesta matriz. Os estudos de Silva et al. (2013a) e Judprasong et al. (2005) avaliaram a solubilidade de $\mathrm{Cu}, \mathrm{Fe} \mathrm{Mn}$ e $\mathrm{Zn}$, e a diálise em fluxo contínuo de $\mathrm{Ca}$, $\mathrm{Fe}, \mathrm{Mg}$, P e Zn, respectivamente, em diferentes matrizes e em materiais de referências certificados de FI. De forma diversa, os estudos de Bosscher et al. (2003), Bosscher et al. (2001), Domínguez et al. (2004), Drago \& Valencia (2004), Laparra et al. (2005), Perales et al. (2007), Frontela et al. (2008) e Aly et al. (2016) focaram em características específicas, avaliando a bioacessibilidade e/ou a biodisponibilidade dos elementos em amostras modificadas em laboratório.

Tabela 2. Estudos envolvendo modelos in vitro para determinação de minerais em FI.

\begin{tabular}{ccccc}
\hline Referência & Elemento estudado & Método & Amostra & Enzimas \\
\hline Aly et al., 2016 & Fe & $\begin{array}{c}\text { Solubilidade e célula } \\
\text { Caco-2 }\end{array}$ & $\begin{array}{c}\text { Amostras preparadas } \\
\text { em laboratório }\end{array}$ & $\begin{array}{c}\text { Pepsina, pancreatina } \\
\text { e sal biliar }\end{array}$ \\
\hline Bermejo et al., 2002 & Fe e Zn & Solubilidade & $\begin{array}{c}\text { Leite materno; 12 FI } \\
\text { comerciais }\end{array}$ & Pepsina, pancreatina \\
\hline Bosscher et al., 2001 & Ca, Fe e Zn & $\begin{array}{c}\text { Dialisabilidade } \\
\text { (diálise em fluxo } \\
\text { contínuo) }\end{array}$ & $\begin{array}{c}\text { Leite materno; FI } \\
\text { preparadas em } \\
\text { Laboratório }\end{array}$ & $\begin{array}{c}\text { Pepsina, pancreatina } \\
\text { e sal biliar }\end{array}$ \\
\hline Bosscher et al., 2003 & Ca, Fe e Zn & $\begin{array}{c}\text { Dialisabilidade } \\
\text { (diálise em fluxo } \\
\text { contínuo) }\end{array}$ & $\begin{array}{c}\text { Leite materno; FI } \\
\text { padrão e especiais } \\
\text { (com espessante) }\end{array}$ & $\begin{array}{c}\text { Pepsina, pancreatina } \\
\text { e sal biliar }\end{array}$ \\
\hline
\end{tabular}


Uma abordagem dos ensaios in vitro para estimar a absorção dos minerais em fórmulas infantis

Fioravanti, M. I. A., \& Morgano, M. A.

Tabela 2. Continuação...

\begin{tabular}{|c|c|c|c|c|c|}
\hline Referência & Elemento estudado & Método & Amostra & Enzimas & Técnica* \\
\hline Silva et al., 2013a & $\mathrm{Cu}, \mathrm{Fe}, \mathrm{Mn}$ e $\mathrm{Zn}$ & Solubilidade & $\begin{array}{l}\text { Matrizes variadas; } \\
\text { material de } \\
\text { referência certificado } \\
\text { FI }\end{array}$ & $\begin{array}{c}\alpha \text {-amilase, Pepsina, } \\
\text { pancreatina e sal } \\
\text { biliar; Ureia; } \\
\text { Glucose; mucina; } \\
\text { alumina }\end{array}$ & ICP-MS \\
\hline $\begin{array}{c}\text { Domínguez et al., } \\
2004\end{array}$ & $\mathrm{Cu}, \mathrm{Fe}$ e $\mathrm{Zn}$ & Dialisabilidade & $\begin{array}{l}\text { FI modificada em } \\
\text { laboratório }\end{array}$ & $\begin{array}{c}\text { Pepsina e } \\
\text { pancreatina }\end{array}$ & ICP OES \\
\hline $\begin{array}{l}\text { Drago \& Valencia, } \\
2004\end{array}$ & $\mathrm{Ca}, \mathrm{Fe}$ e $\mathrm{Zn}$ & Dialisabilidade & $\begin{array}{l}\text { FI preparada em } \\
\text { laboratório }\end{array}$ & $\begin{array}{c}\text { Pepsina, pancreatina } \\
\text { e sal biliar }\end{array}$ & FAAS \\
\hline Frontela et al., 2008 & $\mathrm{Ca}, \mathrm{Fe}$ e $\mathrm{Zn}$ & $\begin{array}{l}\text { Solubilidade e } \\
\text { dialisabilidade }\end{array}$ & $\begin{array}{l}\text { Cereal infantil } \\
\text { reconstituído em FI } \\
\text { ou água }\end{array}$ & $\begin{array}{c}\text { Pepsina, pancreatina } \\
\text { e sal biliar }\end{array}$ & FAAS \\
\hline García et al., 1998 & $\mathrm{Fe}, \mathrm{Cu}$ e $\mathrm{Zn}$ & Dialisabilidade & $\begin{array}{c}6 \text { tipos diferentes de } \\
\text { FI comerciais }(\mathrm{n}= \\
18)\end{array}$ & $\begin{array}{c}\text { Pepsina, pancreatina } \\
\text { e sal biliar }\end{array}$ & $\begin{array}{c}\text { FAAS; ETAAS; } \\
\text { Espectroscopia } \\
\text { UV/visível }\end{array}$ \\
\hline Glahn et al., 1998 & $\mathrm{Fe}$ & Célula Caco-2 & $\begin{array}{l}\text { Leite materno; FI } \\
\text { padrão }\end{array}$ & $\begin{array}{c}\text { Pepsina, pancreatina } \\
\text { e sal biliar }\end{array}$ & $\begin{array}{c}{ }^{59} \mathrm{Fe} \text { em um contador } \\
\text { gama automático }\end{array}$ \\
\hline $\begin{array}{l}\text { Gomez, Perez- } \\
\text { Corona \& Madrid, } \\
2016\end{array}$ & $\mathrm{Zn}$ & $\begin{array}{l}\text { Solubilidade e } \\
\text { Dialisabilidade }\end{array}$ & $\begin{array}{c}6 \text { diferentes FI } \\
\text { comerciais }\end{array}$ & $\begin{array}{c}\alpha \text {-amilase, Pepsina, } \\
\text { pancreatina e sal } \\
\text { biliar }\end{array}$ & $\begin{array}{c}\text { FAAS; } \\
\text { Espectroscopia } \\
\text { UV/visível e ICP- } \\
\text { MS } \\
\end{array}$ \\
\hline Jovaní et al., 2000 & $\mathrm{Ca}, \mathrm{Cu}, \mathrm{Fe}$ e $\mathrm{Zn}$ & Dialisabilidade & $\begin{array}{c}8 \text { diferentes FI } \\
\text { comerciais }\end{array}$ & $\begin{array}{c}\text { Pepsina, pancreatina } \\
\text { e sal biliar }\end{array}$ & FAAS; ETAAS \\
\hline Jovaní et al., 2001 & $\mathrm{Ca}, \mathrm{Fe}$ e $\mathrm{Zn}$ & $\begin{array}{l}\text { Solubilidade, } \\
\text { dialisabilidade e } \\
\text { célula Caco-2 } \\
\end{array}$ & $\begin{array}{l}3 \text { FI especiais } \\
\text { comerciais }\end{array}$ & $\begin{array}{c}\text { Pepsina, pancreatina } \\
\text { e sal biliar }\end{array}$ & AAS \\
\hline $\begin{array}{l}\text { Judprasong et al., } \\
2005\end{array}$ & $\mathrm{Ca}, \mathrm{Fe}, \mathrm{Mg}, \mathrm{P} \mathrm{e} \mathrm{Zn}$ & $\begin{array}{c}\text { Diálise em fluxo } \\
\text { contínuo (método } \\
\text { CFD }^{* *} \text { ) }\end{array}$ & $\begin{array}{c}\text { Matriz variada; } \\
\text { material de } \\
\text { referência certificado } \\
\text { FI }\end{array}$ & $\begin{array}{c}\text { Pepsina, pancreatina } \\
\text { e sal biliar }\end{array}$ & ICP OES \\
\hline $\begin{array}{l}\text { Laparra, Barberá, \& } \\
\text { Farré, } 2005\end{array}$ & $\mathrm{Ca}, \mathrm{Fe}$ e $\mathrm{Zn}$ & $\begin{array}{l}\text { Solubilidade e célula } \\
\text { Caco-2 }\end{array}$ & $\begin{array}{c}\text { FI adaptada em } \\
\text { laboratório }\end{array}$ & $\begin{array}{c}\text { Pepsina, pancreatina } \\
\text { e sal biliar }\end{array}$ & AAS \\
\hline Peña et al., 2004 & $\mathrm{Cu}$ & Solubilidade & $\begin{array}{l}\text { Leite materno; } 1 \mathrm{FI} \\
\text { comercial }\end{array}$ & Pepsina, pancreatina & ETAAS \\
\hline Perales et al., 2005 & $\mathrm{Ca}$ & $\begin{array}{c}\text { Solubilidade, } \\
\text { dialisabilidade e } \\
\text { célula Caco-2 }\end{array}$ & $\begin{array}{l}9 \text { tipos refeições, } 2 \\
\text { FI adaptadas; } 2 \text { FI } \\
\text { comerciais }\end{array}$ & $\begin{array}{c}\text { Pepsina, pancreatina } \\
\text { e sal biliar }\end{array}$ & AAS \\
\hline Perales et al., 2007 & $\mathrm{Fe}$ & $\begin{array}{l}\text { Solubilidade, } \\
\text { dialisabilidade e } \\
\text { célula Caco-2 }\end{array}$ & $\begin{array}{c}\text { Amostras } \\
\text { modificadas em } \\
\text { laboratório }\end{array}$ & $\begin{array}{c}\text { Pepsina, pancreatina } \\
\text { e sal biliar }\end{array}$ & AAS \\
\hline Roig et al., 1999 & $\mathrm{Ca}$ & $\begin{array}{l}\text { Solubilidade e } \\
\text { dialisabilidade }\end{array}$ & $\begin{array}{l}\text { Leite materno; leite } \\
\text { de vaca integral, } 2 \text { FI } \\
\text { comerciais }\end{array}$ & $\begin{array}{c}\text { Pepsina, pancreatina } \\
\text { e sal biliar }\end{array}$ & AAS \\
\hline $\begin{array}{l}\text { Sarriá \& Vaqueiro, } \\
2001\end{array}$ & $\mathrm{Fe}$ e Zn & $\begin{array}{l}\text { Solubilidade e } \\
\text { dialisabilidade }\end{array}$ & $\begin{array}{l}\text { FI comercial em pó e } \\
\text { liquida }\end{array}$ & $\begin{array}{c}\text { Pepsina, pancreatina } \\
\text { e sal biliar }\end{array}$ & AAS \\
\hline
\end{tabular}

*AAS, FAAS: espectrometria de absorção atômica com chama; ICP-MS: espectrometria de massas com fonte de plasma com acoplamento indutivo; ICP OES: espectrometria de emissão ótica com plasma acoplado indutivamente; ETAAS: Espectrometria de absorção atômica com atomização eletrotérmica. ${ }^{* *}$ CFD: do inglês, diálise em fluxo contínuo (continuous-flow dialysis).

$\mathrm{Ca}, \mathrm{Fe}$ e Zn presentes nas FI são os elementos mais estudados quanto a solubilidade, dialisabilidade e biodisponibilidade (célula caco-2). $\mathrm{O} \mathrm{Cu}$ foi avaliado quanto à sua bioacessibilidade nos estudos de García et al. (1998) e de Jovaní et al. (2000), enquanto Peña et al. (2004) desenvolveram um método para determinar sua solubilidade. Não foram encontrados estudos citando a bioacessibilidade ou a biodisponibilidade dos elementos K, Mn, Mg e P, em FI. 
A importância de conhecer o comportamento da matriz e suas variações nos alimentos foi reportada no estudo de Gomez et al. (2016), que compararam a solubilidade e a dialisabilidade do $\mathrm{Zn}$ em diferentes FI comercializadas na Espanha. A fração solúvel de $\mathrm{Zn}$ foi de $30 \%$ a $70 \%$, sendo superiores às porcentagens encontradas no leite de vaca (15-20\%), sugerindo que a suplementação das fórmulas com sais de Zn e o emprego de diferentes tratamentos tecnológicos, como uso de proteína hidrolisada, adição de prebióticos e enriquecimento com minerais, podem favorecer a solubilidade do Zn na FI. Porém, a porcentagem de Zn nas frações dialisadas foi inferior a $11 \%$.

Roig et al. (1999) encontraram uma quantidade de Ca total em leite materno de 29,2 mg/ $100 \mathrm{~mL}$, sendo que, no ensaio de bioacessibilidade, a fração dialisável correspondeu a 13,6\% e a fração solúvel a 55,8\%. Para FI à base de proteína de leite de vaca, foi encontrado um teor total de 42,3 $\mathrm{mg} \mathrm{de} \mathrm{Ca/} 100 \mathrm{~mL}$ e frações dialisável e solúvel de $8,1 \%$ e 11,4\%, respectivamente. Essa tendência de a porcentagem de Ca bioacessível (frações solúvel e dialisável) em FI ser menor em relação ao leite materno também foi observada no estudo de Bosscher et al. (2003).

O leite materno e o leite de vaca apresentam diferenças em suas composições em relação aos tipos e às proporções de proteínas presentes. As FI são elaboradas com uma proporção de proteína do soro/caseína semelhante à encontrada no leite materno maduro (60/40), mas contêm todas as caseínas do leite de vaca (Donovan, 2019). É importante observar que essas diferenças, juntamente com o tamanho das partículas dos sais adicionados e a presença de inibidores (como fitatos), geralmente, são a causa da menor bioacessibilidade do Ca em FI.

As novas tecnologias utilizadas na elaboração das FI para buscar uma aproximação com a biodisponibilidade dos nutrientes presentes no leite materno ficam evidentes no trabalho realizado por Aly et al. (2016). Os autores investigaram a funcionalidade da lactoferrina humana recombinante (rhLf obtida de arroz transgênico) e dos Galacto-oligossacarídeos (GOS) quanto aos efeitos na biodisponibilidade do Fe em FI, concluindo que o rhLf e o GOS são componentes possivelmente adequados para serem incluídos nas FI, pois aumentaram a biodisponibilidade do Fe (de 5 a 7,5 vezes), proporcionando uma melhora no desenvolvimento do lactente.

Ainda existe uma escassez de informações abrangentes sobre a bioacessibilidade e a biodisponibilidade mineral em FI, demandando maiores esforços para entender a real influência dos diversos ingredientes na absorção dos nutrientes, nestes alimentos. Os modelos de digestão in vitro que simulam o sistema gastrointestinal infantil estão aumentando sua aplicabilidade devido a esforços na padronização dos protocolos utilizados, dando destaque aos trabalhos realizados pelo grupo INFOGEST (Minekus et al., 2014; Brodkorb et al., 2019) e Ménard et al., 2018. Contudo, devido às variações e discrepâncias encontradas nestes modelos, ainda há a necessidade de esforços para definir um modelo estático de digestão simples, harmonizado e de consenso (Shani-Levi et al., 2017), principalmente em relação aos minerais.

A bioacessibilidade e a biodisponibilidade dos minerais em FI podem ser afetadas pelos diferentes tipos de tecnologias empregadas durante sua elaboração, como tipo de proteína (hidrolisada, proteína isolada, entre outras), adição de prebióticos, fortificações e as concentrações dos elementos em cada FI. Portanto, em busca de atingir o mesmo nível de absorção dos nutrientes fornecidos pelo leite materno, os ensaios in vitro apresentam potenciais quanto à melhoria as FI disponíveis no comércio de um modo simples e rápido, conforme as condições gastrointestinais específicas dos lactentes são mais bem compreendidas.

\section{Conclusão}

As fórmulas infantis são, sem dúvida, o melhor substituinte do leite materno para lactentes, sendo que sua composição é constantemente melhorada e controlada pelos órgãos de regulamentação e pela própria indústria devido à importância deste alimento no crescimento e no desenvolvimento das crianças. Os modelos de digestão in vitro possibilitam entender a influência dos diferentes ingredientes na absorção dos nutrientes, 
contribuindo para que as fórmulas infantis proporcionem, de forma cada vez mais semelhante, a bioacessibilidade e biodisponibilidade presentes no leite materno.

Em relação às diferentes faixas etárias, há uma tendência de maiores concentrações dos elementos nas fórmulas para a faixa de 6 a 12 meses que naquelas para menores de 6 meses. Estas diferenças na composição permitem uma ingestão mais equilibrada de nutrientes nas diferentes fases do lactente, de forma semelhante às mudanças de composição que ocorrem naturalmente no leite materno. Portanto, as fórmulas infantis com faixas etárias definidas é um avanço nutricional para a alimentação no início da vida. Ciente de que a composição delas é frequentemente sujeita a alterações e melhorias, incluindo o aumento das concentrações dos elementos, faz-se necessário um contínuo controle destes alimentos destinados a lactentes e crianças.

\section{Agradecimentos}

Os autores agradecem à Fundação de Amparo à Pesquisa do Estado de São Paulo (FAPESP 2017/11334-8) e ao Conselho Nacional de Desenvolvimento Científico e Tecnológico (CNPq, 303142/2017-0) pelo apoio financeiro. O presente trabalho foi realizado com apoio da Coordenação de Aperfeiçoamento de Pessoal de Nível Superior - Brasil (CAPES) - Código de Financiamento 001.

\section{Referências}

Alegría-Torán, A., Barbera-Sáez, R., \& Cilla-Tatay, A. (2015). Bioavailability of minerals in foods. In M. Guardia \& S. Garrigues (Eds.), Handbook of mineral elements in food. (Chap 3, pp. 41-67). Valencia: John Wiley \& Sons.

Aly, E., López-Nicolás, R., Darwish, A. A., Frontela-Saseta, C., \& Ros-Berruezo, G. (2016). Supplementation of infant formula with recombinant human lactoferrin and/or galactooligosaccharides increases iron bioaccessibility as measured by ferritin formed in Caco-2 cell model. Food Research International, 89(3), 1048-1055. http://dx.doi.org/10.1016/j.foodres.2016.08.030

Barciela-Alonso, M. C., \& Bermejo-Barrera, P. (2015). Variation of food mineral content during industrial and culinary processing. In M. Guardia \& S. Garrigues (Eds.), Handbook of mineral elements in food. (Chap 8, pp. 163-176). Valencia: John Wiley \& Sons.

Bermejo, P., Peña, E. M., Domínguez, R., Bermejo, A., Cocho, J. A., \& Fraga, J. M. (2002). Iron and zinc in hydrolised fractions of human milk and infant formula using an in vitro method. Food Chemistry, 77(3), 361-369. http://dx.doi.org/10.1016/S03088146(02)00111-5

Bosscher, D., Van Caillie-Bertrand, M., Robberecht, H., Van Dyck, K., Van Cauwenbergh, R., \& Deelstra, H. (2001). In vitro availability of calcium, iron, and zinc from first-age infant formulae and human milk. Journal of Pediatric Gastroenterology and Nutrition, 32(1), 54-58. PMid:11176326. http://dx.doi.org/10.1097/00005176-200101000-00016

Bosscher, D., Van Caillie-Bertrand, M., Van Cauwenbergh, R., \& Deelstra, H. (2003). Availabilities of calcium, iron, and zinc from dairy infant formula is affected by soluble dietary fibers and modified starch fractions. Nutrition, 19(7-8), 641-645. PMid:12831951. http://dx.doi.org/10.1016/S0899-9007(03)00063-7

Brasil. Ministério da Saúde. Agência Nacional de Vigilância Sanitária - ANVISA. (2011a, setembro 19). Dispõe sobre o regulamento técnico de compostos de nutrientes para alimentos destinados a lactentes e a crianças de primeira infância (Resolução - RDC n 42, de 19 de setembro de 2011). Diário Oficial [da] República Federativa do Brasil, Brasília. Recuperado em 20 de setembro de 2019, de https://portal.anvisa.gov.br.

Brasil. Ministério da Saúde. Agência Nacional de Vigilância Sanitária - ANVISA. (2011b, setembro 19). Dispõe sobre o regulamento técnico para fórmulas infantis para lactentes (Resolução - RDC n 43, de 19 de setembro de 2011). Diário Oficial [da] República Federativa do Brasil. Brasília. Recuperado em 20 de setembro de 2019, de https://portal.anvisa.gov.br.

Brasil. Ministério da Saúde. Agência Nacional de Vigilância Sanitária - ANVISA. (2011c, setembro 19). Dispõe sobre o regulamento técnico para fórmulas infantis de seguimento para lactentes e crianças de primeira infância (Resolução - RDC $n^{\circ}$ 44, de 19 de setembro de 2011). Diário Oficial [da] República Federativa do Brasil. Brasília. Recuperado em 20 de setembro de 2019, de https://portal.anvisa.gov.br.

Brasil. Ministério da Saúde. Agência Nacional de Vigilância Sanitária - ANVISA. (2011d, setembro 19). Dispõe sobre o regulamento técnico para fórmulas infantis para lactentes destinadas a necessidades dietoterápicas específicas e fórmulas infantis de seguimento para lactentes e crianças de primeira infância destinadas a necessidades dietoterápicas específicas (Resolução - RDC n 45, de 19 de setembro de 2011). Diário Oficial [da] República Federativa do Brasil. Brasília. Recuperado em 20 de setembro de 2019, de https://portal.anvisa.gov.br.

Brodkorb, A., Egger, L., Alminger, M., Alvito, P., Assunção, R., Ballance, S., Bohn, T., Bourlieu-Lacanal, C., Boutrou, R., Carrière, F., Clemente, A., Corredig, M., Dupont, D., Dufour, C., Edwards, C., Golding, M., Karakaya, S., Kirkhus, B., Le Feunteun, S., Lesmes, U., Macierzanka, A., Mackie, A. R., Martins, C., Marze, S., McClements, D. J., Ménard, O., Minekus, M., Portmann, R., Santos, C. N., Souchon, I., Singh, R. P., Vegarud, G. E., Wickham, M. S. J., Weitschies, W., \& Recio, I. (2019). INFOGEST static in vitro simulation of gastrointestinal food digestion. Nature Protocols, 14(4), 991-1014. PMid:30886367. http://dx.doi.org/10.1038/s41596-018-0119-1 
Domínguez, R., Barreiro, T., Sousa, E., Bermejo, A., Cocho, J. A., Fraga, J. M., \& Bermejo, P. (2004). Study of the effect of different iron salts used to fortify infant formulas on the bioavailability of trace elements using ICP-OES. International Dairy Journal, 14(12), 1081-1087. http://dx.doi.org/10.1016/j.idairyj.2004.03.011

Donovan, S. M. (2019). Human milk proteins: Composition and physiological significance. Nestlé Nutrition Institute Workshop Series, 90, 93-101. PMid:30865978. http://dx.doi.org/10.1159/000490298

Drago, S. R., \& Valencia, M. E. (2004). Influence of components of infant formulas on in vitro iron, zinc, and calcium availability. Journal of Agricultural and Food Chemistry, 52(10), 3202-3207. PMid:15137876. http://dx.doi.org/10.1021/jf035191e

Drapala, K. P., Mulvihill, D. M., \& O'Mahony, J. A. (2018). Improving the oxidative stability of model whey protein hydrolysatebased infant formula emulsions with lecithin. International Journal of Dairy Technology, 71(4), 966-974. http://dx.doi.org/10.1111/1471-0307.12538

Etcheverry, P., Grusak, M. A., \& Fleige, L. E. (2012). Application of in vitro bioaccessibility and bioavailability methods for Calcium, carotenoids, Folate, Iron, Magnesium, Polyphenols, Zinc, and vitamins B6, B12, D, and E. Frontiers in Physiology, 3, 317. PMid:22934067. http://dx.doi.org/10.3389/fphys.2012.00317

European Commission - EC. (2006). Directiva 2006/141/CE da Comissão de 22 de Dezembro de 2006, relativa às fórmulas para lactentes e fórmulas de transição e que altera a Directiva 1999/21/CE, L401. Official Journal of the European Union, Bruxelas.

Fei, P., Feng, H., Wang, Y., Kang, H., Xing, M., Chang, Y., Guo, L., \& Chen, J. (2020). Amaranthus tricolor crude extract inhibits Cronobacter sakazakii isolated from powdered infant formula. Journal of Dairy Science, 103(11), 9969-9979. PMid:32861498. http://dx.doi.org/10.3168/jds.2020-18480

Fioravanti, M. I. A., Milani, R. F., Paiva, E. L., \& Morgano, M. A. (2020a). Simple and fast ultrasound-assisted method for mineral content and bioaccessibility study in infant formula by ICP OES. Analytical Methods, 12(25), 3225-3234. PMid:32930185. http://dx.doi.org/10.1039/D0AY00867B

Fioravanti, M. I. A., Milani, R. F., Paiva, E. L., \& Morgano, M. A. (2020b). Influence of various ingredients on mineral bioaccessibility in infant formula and whole milk. International Dairy Journal, 110, 104808. http://dx.doi.org/10.1016/j.idairyj.2020.104808

Food and Agriculture Organization of the United Nations - FAO. World Health Organization - FAO/WHO. (2016). Standard for infant formula and formulas for special medical purposes intended for infants. (Codex Stan 72 - 1981) Rome: FAO.

Frontela, C., Haro, J. F., Ros, G., \& Martínez, C. (2008). Effect of dephytinization and follow-on formula addition on in vitro iron, calcium, and zinc availability from infant cereals. Journal of Agricultural and Food Chemistry, 56(10), 3805-3811. PMid:18433137. http://dx.doi.org/10.1021/jf073424m

Fu, S., Jiang, Y., Qin, X., Yang, T., Chen, S., Yang, X., Zhang, W., Qu, Y., \& Man, C. (2020). Electricity-free amplification and visual detection of Cronobacter species in powdered infant formula. Journal of Dairy Science, 103(8), 6882-6893. PMid:32505404. http://dx.doi.org/10.3168/jds.2019-17661

García, R., Alegría, A., Barberá, R., Farre, R., \& Lagarda, M. J. (1998). Dialyzability of iron, zinc, and copper of different types of infant formulas marketed in Spain. Biological Trace Element Research, 65(1), 7-17. PMid:9877533. http://dx.doi.org/10.1007/BF02784110

Glahn, R. P., Lai, C., Hsu, J., Thompson, J. F., Guo, M., \& Van Campen, D. R. (1998). Decreased Citrate Improves iron availability from infant formula: Application of an in vitro digestion/caco-2 cell culture model. The Journal of Nutrition, 128(2), 257-264. PMid:9446853. http://dx.doi.org/10.1093/jn/128.2.257

Gomez, B. G., Perez-Corona, M. T., \& Madrid, Y. (2016). Availability of Zinc from infant formula by in vitro methods (solubility and dialyzability) and size-exclusion chromatography coupled to inductively coupled plasma-mass spectrometry. Journal of Dairy Science, 99(12), 9405-9414. PMid:27771091. http://dx.doi.org/10.3168/jds.2016-11405

Hur, S. J., Lim, B. O., Decker, E. A., \& McClements, D. J. (2011). In vitro human digestion models for food applications. Food Chemistry, 125(1), 1-12. http://dx.doi.org/10.1016/j.foodchem.2010.08.036

Jovaní, M., Alegría, A., Barberá, R., Farré, R., Lagarda, M. J., \& Clemente, G. (2000). Effect of proteins, phytates, ascorbic acid and citric acid on dialysability of calcium, iron, zinc and copper in soy-based infant formulas. Die Nahrung, 44(2), 114-117. PMid:10795579. http://dx.doi.org/10.1002/(SICI)1521-3803(20000301)44:2<114::AID-FOOD114>3.0.CO;2-N

Jovaní, M., Barberá, R., Farré, R., \& Martín de Aguilera, E. (2001). Calcium, iron, and zinc uptake from digests of infant formulas by caco-2 cells. Journal of Agricultural and Food Chemistry, 49(7), 3480-3485. PMid:11453795. http://dx.doi.org/10.1021/jf010106t

Judprasong, K., Ornthai, M., Siripinyanond, A., \& Shiowatana, J. (2005). A continuous-flow dialysis system with inductively coupled plasma optical emission spectrometry for in vitro estimation of bioavailability. Journal of Analytical Atomic Spectrometry, 20(11), 1191. http://dx.doi.org/10.1039/b508639f

Laparra, J. M., Barberá, R., \& Farré, R. (2005). Effect of enzyme amounts used in gastrointestinal digestion upon solubility and caco-2 cell uptake assays of minerals from infant formulas. Food Science and Technology International, 11(6), 425-431. http://dx.doi.org/10.1177/1082013205060195

Li, S., Sijtsema, S. J., Kornelis, M., Liu, Y., \& Li, S. (2019). Consumer confidence in the safety of milk and infant milk formula in China. Journal of Dairy Science, 102(10), 8807-8818. PMid:31421878. http://dx.doi.org/10.3168/jds.2019-16638

Lönnerdal, B., \& Hernell, O. (2016). An opinion on "Staging" of infant formula: A developmental perspective on infant feeding. Journal of Pediatric Gastroenterology and Nutrition, 62(1), 9-21. PMid:25844707. http://dx.doi.org/10.1097/MPG.0000000000000806 
Lucas-González, R., Viuda-Martos, M., Pérez-Alvarez, J. A., \& Fernández-López, J. (2018). In vitro digestion models suitable for foods: Opportunities for new fields of application and challenges. Food Research International, 107, 423-436. PMid:29580504. http://dx.doi.org/10.1016/j.foodres.2018.02.055

Luten, J., Crews, H., Flynn, A., Van Dael, P., Kastenmayer, P., Hurrell, R., Deelstra, H., Shen, L., Fairweather-Tait, S., Hickson, K., Farré, R., Schlemmer, U., \& Frohlich, W. (1996). Interlaboratory trial on the determination of the in vitro iron dialyzability from food. Journal of the Science of Food and Agriculture, 72(4), 415-424. http://dx.doi.org/10.1002/(SICI)10970010(199612)72:4<415::AID-JSFA675>3.0.CO;2-X

Martin, C. R., Ling, P. R., \& Blackburn, G. L. (2016). Review of infant feeding: Key features of breast milk and infant formula. Nutrients, 8(5), 279. PMid:27187450. http://dx.doi.org/10.3390/nu8050279

Martínez, M. A., Castro, I., Rovira, J., Ares, S., Rodríguez, J. M., Cunha, S. C., Casal, S., Fernandes, J. O., Schuhmacher, M., \& Nadal, M. (2019). Early-life intake of major trace elements, bisphenol A, tetrabromobisphenol A and fatty acids: Comparing human milk and commercial infant formulas. Environmental Research, 169, 246-255. PMid:30476748. http://dx.doi.org/10.1016/j.envres.2018.11.017

Ménard, O., Bourlieu, C., De Oliveira, S. C., Dellarosa, N., Laghi, L., Carrière, F., Capozzi, F., Dupont, D., \& Deglaire, A. (2018). A first step towards a consensus static in vitro model for simulating full-term infant digestion. Food Chemistry, 240, 338-345. PMid:28946281. http://dx.doi.org/10.1016/j.foodchem.2017.07.145

Miller, D. D., Schricker, B. R., Rasmussen, R. R., \& Van Campen, D. (1981). An in vitro method for estimation of iron availability from meals. The American Journal of Clinical Nutrition, 34(10), 2248-2256. PMid:6794346. http://dx.doi.org/10.1093/ajcn/34.10.2248

Minekus, M., Alminger, M., Alvito, P., Ballance, S., Bohn, T., Bourlieu, C., Carrière, F., Boutrou, R., Corredig, M., Dupont, D., Dufour, C., Egger, L., Golding, M., Karakaya, S., Kirkhus, B., Le Feunteun, S., Lesmes, U., Macierzanka, A., Mackie, A., Marze, S., McClements, D. J., Ménard, O., Recio, I., Santos, C. N., Singh, R. P., Vegarud, G. E., Wickham, M. S. J., Weitschies, W., \& Brodkorb, A. (2014). A standardised static in vitro digestion method suitable for food: An international consensus. Food \& Function, 5(6), 1113-1124. PMid:24803111. http://dx.doi.org/10.1039/C3FO60702J

Moreda-Piñeiro, J., Moreda-Piñeiro, A., Romarís-Hortas, V., Moscoso-Pérez, J., López-Mahía, P., Muniategui-Lorenzo, S., Bermejo-Barrera, P., \& Prada-Rodríguez, D. (2011). In vivo and in vitro testing to assess the bioaccessibility and the bioavailability of arsenic, selenium and mercury species in food samples. Trends in Analytical Chemistry, 30(2), 324-345. http://dx.doi.org/10.1016/j.trac.2010.09.008

Moreira, L. C. Q., Oliveira, E. B. E., Lopes, L. H. K., Bauleo, M. E., \& Sarno, F. (2019). Introduction of complementary foods in infants. Einstein, 17(3), eAO4412. PMid:31116228. http://dx.doi.org/10.31744/einstein journal/2019AO4412

Padovani, R. M., Amaya-Farfán, J., Colugnati, F. A. B., \& Domene, S. M. A. (2006). Dietary reference intakes: Aplicabilidade das tabelas em estudos nutricionais. Revista de Nutrição, 19(6), 741-760. http://dx.doi.org/10.1590/S1415-52732006000600010

Peña, E., Domínguez, R., Bermejo, A., Cocho, J. A., Fraga, J. M., \& Bermejo, P. (2004). Enzymolysis approach to compare Cu availability from human milk and infant formulas. Journal of Agricultural and Food Chemistry, 52(15), 4887-4892. PMid:15264930. http://dx.doi.org/10.1021/jf030781y

Perales, S., Barberá, R., Lagarda, M. J., \& Farré, R. (2005). Bioavailability of calcium from milk-based formulas and fruit juices containing milk and cereals estimated by in vitro methods (solubility, dialyzability, and uptake and transport by caco-2 cells). Journal of Agricultural and Food Chemistry, 53(9), 3721-3726. PMid:15853426. http://dx.doi.org/10.1021/jf047977y

Perales, S., Barberá, R., Lagarda, M. J., \& Farré, R. (2007). Availability of iron from milk-based formulas and fruit juices containing milk and cereals estimated by in vitro methods (solubility, dialysability) and uptake and transport by caco-2 cells. Food Chemistry, 102(4), 1296-1303. http://dx.doi.org/10.1016/j.foodchem.2006.07.019

Pires, R. P. S., Cappato, L. P., Guimarães, J. T., Rocha, R. S., Silva, R., Balthazar, C. F., Freitas, M. Q., Silva, P. H. F., Neto, R. P. C., Tavares, M. I. B., Granato, D., Raices, R. S. L., Silva, M. C., \& Cruz, A. G. (2020). Ohmic heating for infant formula processing: Evaluating the effect of different voltage gradient. Journal of Food Engineering, 280, 109989. http://dx.doi.org/10.1016/j.jfoodeng.2020.109989

Poitevin, E. (2016). Official methods for the determination of minerals and trace elements in infant formula and milk products: A review. Journal of AOAC International, 99(1), 42-52. PMid:26821839. http://dx.doi.org/10.5740/jaoacint.15-0246

Roig, M. J., Alegría, A., Barberá, R., Farré, R., \& Lagarda, M. J. (1999). Calcium bioavailability in human milk, cow milk and infant formulas: Comparison between dialysis and solubility methods. Food Chemistry, 65(3), 353-357. http://dx.doi.org/10.1016/S0308-8146(98)00232-5

Sarriá, B., \& Vaquero, M. P. (2001). Zinc and iron bioavailability in a powder or in-bottle-sterilized infant formula estimated by in vitro and in suckling rats. The Journal of Nutritional Biochemistry, 12(5), 266-273. PMid:11382544. http://dx.doi.org/10.1016/S0955-2863(01)00138-3

Shani-Levi, C., Alvito, P., Andrés, A., Assunção, R., Barberá, R., Blanquet-Diot, S., Bourlieu, C., Brodkorb, A., Cilla, A., Deglaire, A., Denis, S., Dupont, D., Heredia, A., Karakaya, S., Giosafatto, C. V. L., Mariniello, L., Martins, C., Ménard, O., El, S. N., Vegarud, G. E., Ulleberg, E., \& Lesmes, U. (2017). Extending in vitro digestion models to specific human populations: Perspectives, practical tools and bio-relevant information. Trends in Food Science \& Technology, 60, 52-63. http://dx.doi.org/10.1016/j.tifs.2016.10.017

Silva, E. N., Leme, A. B. P., Cidade, M., \& Cadore, S. (2013a). Evaluation of the bioaccessible fractions of Fe, Zn, Cu and Mn in baby foods. Talanta, 117, 184-188. PMid:24209328. http://dx.doi.org/10.1016/j.talanta.2013.09.008

Silva, S. V., Mattanna, P., Bizzi, C. A., Richards, N. S. P. S., \& Barin, J. S. (2013b). Evaluation of the mineral content of infant formulas consumed in Brasil. Journal of Dairy Science, 96(6), 3498-3505. PMid:23608492. http://dx.doi.org/10.3168/jds.2012-6268 
U.S. Food and Drug Administration - FDA. (1985). Nutrient Requerimentes. (Part 107 Infant Formula, Subpart D - 107.100) Silver Spring, MD: U.S Food and Drug Administration. Recuperado em 01 de outubro de 2019, de https://www.accessdata.fda.gov/scripts/cdrh/cfdocs/cfCFR/CFRSearch.cfm?fr=107.10

Zou, L., Pande, G., \& Akoh, C. C. (2016). Infant formula fat analogs and human milk fat: New focus on infant developmental needs. Annual Review of Food Science and Technology, 7(1), 139-165. PMid:26934172. http://dx.doi.org/10.1146/annurevfood-041715-033120

Funding: Ministério da Ciência, Tecnologia e Inovação, Conselho Nacional de Desenvolvimento Científico e Tecnológico (CNPq) (303142/2017-0). Coordenação de Aperfeiçoamento de Pessoal de Nível Superior- Brasil (CAPES)

(Código de Financiamento 001). Fundação de Amparo à Pesquisa do Estado de São Paulo (FAPESP) (2017/11334-8). 